\title{
Ruolo diagnostico, prognostico e predittivo di risposta del NETest nelle neoplasie neuroendocrine
}

\author{
Antongiulio Faggiano ${ }^{1}$. Valentina Di Vito ${ }^{1} \cdot$ Roberta Centello $^{1} \cdot$ Franz Sesti $^{1} \cdot$ Giulia Puliani $^{1} \cdot$ Tiziana Feola $^{1}$. \\ Elisa Giannetta ${ }^{1}$
}

Accettato: 18 giugno 2020 / Pubblicato online: 20 novembre 2020

(c) The Author(s) 2020

Sommario Il NETest è una metodica di biologia molecolare e, in particolare, di biopsia liquida, applicata alle neoplasie neuroendocrine (NEN), che si propone come nuovo biomarcatore altamente sensibile e specifico. Il NETest consente una sorta di gene signature del tumore, definendone il profilo trascrizionale mRNA, estratto dal sangue periferico. L'applicazione pratica è nella diagnosi, dove il NETest sembra identificare anche piccoli tumori localizzati, nella definizione prognostica, con l'identificazione dei tumori con maggiore tendenza alla progressione e alla recidiva post-chirurgica, nella riposta ai trattamenti, con l'identificazione precoce di progressione nel corso di terapie antitumorali. A fronte di risultati iniziali estremamente promettenti, il NETest necessita di una conferma su larga scala, in ampie casistiche multicentriche.

Parole chiave NETest - Biopsia liquida - Diagnosi · Fattori prognostici $\cdot$ Fattori predittivi di risposta $\cdot$ Neoplasie neuroendocrine

\section{Introduzione}

\section{Le neoplasie neuroendocrine}

Le neoplasie neuroendocrine (NEN) sono un gruppo eterogeneo di tumori che originano dal sistema neuroendocrino. Esprimono marcatori di differenziazione neuroendocrina, come Cromogranina A, sinaptofisina ed enolasi neuronospecifica (NSE), nonché ormoni e fattori di trascrizione tissutali specifici [1]. L'eterogeneità delle NEN dipende dalla natura del sistema neuroendocrino, costituito da cellule e tessuti differenti ma con caratteristiche comuni, diffuse in diversi organi e apparati, in particolare a livello di polmoni, tratto gastrointestinale e pancreas [1].

A causa dell'eterogeneità delle NEN, sono stati adottati vari criteri per la loro classificazione. Nel 2010 sia l'OMS che l'American Joint Cancer Committee (AJCC) hanno introdotto i fondamenti della moderna classificazione: il termine NEN include le forme a basso grado, i cosiddetti tumori neuroendocrini (NET), e quelle ad alto grado, i carcinomi neuroendocrini (NEC). I NET presentano morfologia ben differenziata e valori bassi-intermedi di conta mitotica e/o indice Ki-67, mentre i NEC presentano morfologia scarsamente differenziata ed elevati indici proliferativi [2]. Le nuove classificazioni WHO 2017 e 2019 hanno ridefinito la classificazione, suddividendo i NET in G1, G2 e G3, con indice proliferativo crescente, mentre i NEC presentano un'unica categoria a elevata proliferazione [3].

Il perfezionamento della classificazione istologica e del sistema di stadiazione, basato sulla dimensione del tumore/invasione locale, coinvolgimento linfonodale e metastasi a distanza (TNM), ha permesso una migliore caratterizzazione dei tumori, con una previsione prognostica più efficace e indicazioni utili alla scelta terapeutica $[4,5]$. Le strategie terapeutiche attualmente disponibili includono, oltre alla 
Tabella 1 Biomarcatori attualmente in uso nelle neoplasie neuroendocrine

\begin{tabular}{ll}
\hline Marcatore & Indicazione clinica \\
\hline $\begin{array}{l}\text { Marcatori aspecifici } \\
\text { Cromogranina A (CgA) }\end{array}$ & \\
Enolasi Neurone Specifica (NSE) & Tutti i tipi di neoplasie neuroendocrine \\
Polipeptide pancreatico & Carcinoma neuroendocrino, tumore neuroendocrino metastatico, carcinoide atipico \\
Marcatori specifici & Tumore neuroendocrino pancreatico \\
Acido 5-idrossi-indolacetico urinario (5-HIAA) & \\
Gastrina & Tumore neuroendocrino - Sindrome da carcinoide \\
Insulina, C peptide, glicemia & Tumore neuroendocrino duodeno-pancreatico - Sindrome di Zollinger Ellison \\
Glucagone, VIP, somatostatina & Tumore neuroendocrino pancreatico - Sindrome ipoglicemica iperinsulinemica \\
Metanefrine, normetanefrine, 3-metossitiramina & Tumore neuroendocrino pancreatico \\
Calcitonina & Feocromocitomi/paragangliomi \\
NTpro-BPN & Carcinoma midollare, altri tumori neuroendocrini (pancreas, laringe, ecc.) \\
PTH, calcemia, ACTH, cortisolo, GH, IGF1 & Cardiopatia da sindrome da carcinoide come marcatore di disfunzione ventricolare \\
\hline
\end{tabular}

chirurgia, analoghi della somatostatina (SSA), terapia radiorecettoriale (PRRT), terapie a bersaglio molecolare (everolimus, sunitinib), chemioterapia (cisplatino, etoposide, temozolomide, capecitabina), radioterapia e trattamenti ablativi locoregionali $[4,5]$.

Nell'era della medicina di precisione, l'eterogeneità delle NEN rappresenta una sfida clinica significativa, perché rende difficile prevederne la prognosi e ottimizzarne il trattamento [6]. Tra le questioni irrisolte nella gestione delle NEN occupa un piano di rilievo l'assenza di efficaci biomarcatori di diagnosi, prognosi e predittivi di risposta ai trattamenti.

\section{Limiti dei biomarcatori attuali}

Le NEN sintetizzano e secernono vari ormoni, amine e peptidi che possono essere utilizzati come biomarcatori. Questi vengono suddivisi in "aspecifici", comuni a tutte le NEN, e "specifici" di singoli istotipi tumorali e ben definiti fenotipi clinici [7]. Attualmente i biomarcatori monoanalitici utilizzati (Tabella 1) presentano molti limiti in termini di variabilità delle misurazioni, scarsa sensibilità, specificità e potere predittivo. Ciò riflette sia l'eterogeneità che la complessità biologica di questi tumori. Pertanto, sebbene utilizzati nella pratica clinica sia nella diagnosi che nel follow-up, raramente rappresentano una guida nel processo decisionale di queste neoplasie [7, 8].

\section{Marcatori aspecifici}

La Cromogranina $\mathrm{A}(\mathrm{CgA})$ è una glicoproteina presente nei granuli densi secretori delle cellule neuroendocrine ed è il biomarcatore neuroendocrino più utilizzato, correlando con la massa tumorale, soprattutto epatica, e con la sopravvivenza del paziente. Tuttavia, non esiste un dosaggio standardizzato e ci sono ampie variazioni nelle misurazioni effettuate nei diversi laboratori, in base agli anticorpi utilizzati per il dosaggio. La CgA ha una sensibilità del 60-90\% e una specificità molto variabile tra il 10 e il $96 \%$, a causa dei falsi positivi dovuti a differenti condizioni sia patologiche (insufficienza renale o cardiaca, epatopatia cronica, gastrite cronica atrofica, malattie infiammatorie croniche dell'intestino, tumori non neuroendocrini) che farmacologiche (inibitori di pompa protonica) [9].

L'enolasi neurone-specifica (NSE) è espressa nei neuroni e nelle cellule neuroendocrine e può essere indicativa di tumori derivati da questi tipi cellulari. L'NSE non è in grado di differenziare tra diversi sottotipi di NEN, ma elevati livelli di questo enzima sono associati alle neoplasie scarsamente differenziate. L'NSE non è comunemente usato nella pratica clinica in quanto non offre alcun vantaggio particolare rispetto alla $\mathrm{CgA}$ e ha molti limiti, sia di misurazione che di accuratezza diagnostica [8].

Il polipeptide pancreatico (PP) è prodotto dalle cellule neuroendocrine del pancreas e del colon. È stato usato come biomarcatore non specifico, ma non sembra correlare con alcun aspetto specifico delle NEN, pertanto la sua utilità clinica è limitata [8].

\section{Marcatori specifici}

I principali biomarcatori tumorali associati alle differenti sindromi endocrine sono riassunti in Tabella 1.

L'Acido 5-idrossi-indolacetico urinario (5-HIAA) è il secondo marcatore più esplorato nell'ambito delle NEN. Presenta una sensibilità del 5-50\% (65-85\% nei NET ileali) e una specificità quasi del $100 \%$ nell'identificare un tumore serotonina-secernente [7]. L'accuratezza di questo marcatore è elevata quando il valore è almeno doppio rispetto al cut-off massimo, ma necessita di conferma su più campioni 
urinari e dell'esclusione di numerosi fattori interferenti, dietetici (caffeina, teina, banane, banane, pompelmo, kiwi, avocado, more, prugne rosse, frutta secca, melanzane, pomodori, cioccolato) e farmacologici (FANS, antidepressivi triciclici, fenotiazina, reserpina, propanololo, L-DOPA, I-MAO, 5-fluorouracile). Il 5-HIAA correla solo debolmente con la gravità della patologia, e ciò ne limita l'uso nello screening e nella diagnosi [8]. L'NT-proBNP è utilizzato come marcatore di disfunzione ventricolare in pazienti con cardiopatia da sindrome da carcinoide.

La gastrina è il biomarcatore specifico della sindrome di Zollinger-Ellison (ZES) con una sensibilità di circa il 100\% e una bassa specificità. Analogamente alla $\mathrm{CgA}$, la bassa specificità è dovuta a numerose condizioni sia patologiche (insufficienza renale, gastrite cronica atrofica, infezione da Helicobacter pylori, sindrome dell'intestino corto) che farmacologiche (inibitori di pompa protonica, $\mathrm{H} 2$ antagonisti) responsabili di aumentate concentrazioni ematiche [9]. L'insulina è il biomarcatore specifico dell'ipoglicemia iperinsulinemica da insulinoma, in associazione a ipoglicemia ed elevati valori di C-peptide. Presenta una sensibilità del 100\% e una bassa specificità [9]. Livelli di insulina inappropriati si riscontrano anche nella nesidioblastosi, nelle sindromi ipoglicemiche autoimmuni, nelle ipoglicemie farmacologiche. Il glucagone, la somatostatina, il VIP sono ulteriori biomarcatori di specifiche sindromi endocrine correlate alle NENs, generalmente causate da rari NET pancreatici [7].

Le normetanefrine e le metanefrine plasmatiche o urinarie e la 3-metossitiramina plasmatica, sono utilizzate nella diagnosi e nel monitoraggio dei feocromocitomi/paragangliomi. Il dosaggio di questi marcatori richiede l'esclusione di fattori dietetici (caffeina, teina, banane, kiwi, avocado, frutta secca, cioccolato, vaniglia), voluttuari (nicotina, alcol) e farmacologici (FANS, antidepressivi triciclici, litio, reserpina, clonidina, furosemide, felodipina, L-DOPA, I-MAO, nitroglicerina, teofillina, fenotiazina, desametasone) che possono interferire con il dosaggio [10].

La calcitonina è invece il marcatore suggestivo di carcinoma midollare della tiroide ma elevati livelli possono essere riscontrati anche in NEN di altra sede, una volta esclusi anche in questo caso fattori fisiologici, patologici e farmacologici interferenti con il dosaggio [11].

Infine, ulteriori ormoni possono essere sintetizzati e secreti nell' ambito di una sindrome paraneoplastica soprattutto nelle NEN polmonari (carcinoidi tipici) e pancreatiche (NET G2), in particolare in caso di ricorrenza o progressione di malattia [7].

\section{La biopsia liquida in oncologia}

La biopsia liquida è una metodica di nuova generazione che utilizza un campione di sangue al fine di identificare e caratterizzare la neoplasia del paziente [12]. Con il termine

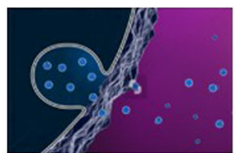

Esosomi
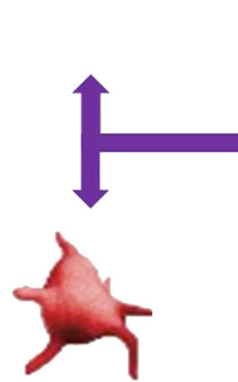

Piastrine

educate dal tumore (TEPS)

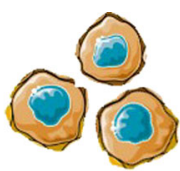

Cellule tumorali circolanti

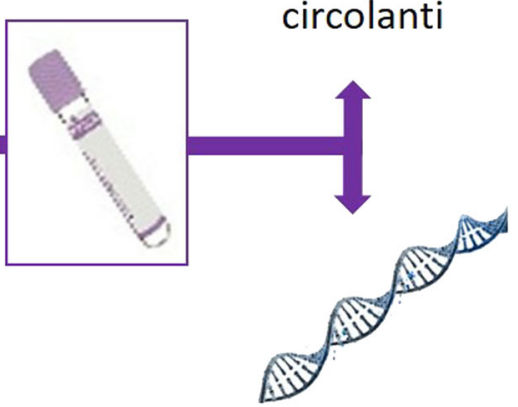

Acidi nucleici
Fig. 1 Biopsia liquida: materiale biologico di partenza

biopsia liquida ci si riferiva inizialmente alla ricerca di cellule tumorali circolanti (CTC); attualmente il concetto è stato ampliato anche all'analisi di acidi nucleici, esosomi o piastrine educate dal tumore (tumor educated platelets, TEPs) [13], come riassunto in Figura 1.

I vantaggi della biopsia liquida sono molteplici. In primis, si tratta di una procedura semplice e gravata da bassi rischi per il paziente: questo rende l'esame eseguibile anche in pazienti ad alto rischio o in cui il tumore si trovi in sedi difficilmente bioptizzabili [13]. Secondo, la procedura è ripetibile e, come tale, si presta per l'individuazione di possibili cloni tumorali divenuti più aggressivi (ad esempio durante l'impiego di farmaci chemioterapici), permettendo un rapido adeguamento della terapia [14]. Terzo, in caso di pazienti clinicamente in remissione, la positività della biopsia liquida potrebbe permettere di evidenziare pazienti a rischio di recidiva (malattia minima residua) [15]. Infine, molti tumori, tra cui le NEN, sono caratterizzati da una marcata eterogeneità, per cui il campione bioptico di una localizzazione tumorale potrebbe non essere esemplificativo di tutte le lesioni del singolo paziente, mentre la biopsia liquida riflette meglio le varie localizzazioni di malattia.

La prima identificazione di CTC risale al 1869 [16] e attualmente la loro valutazione trova un impiego clinico in vari istotipi tumorali tra cui i tumori prostatici, ovarici, polmonari, mammari [12]. Una volta isolate, le CTC sono utilizzate per l'esecuzione di analisi citologiche e molecolari al fine di ottenere informazioni cliniche ma anche di ampliare la conoscenza dei vari processi implicati nella cancerogenesi [17]. Inoltre, l'identificazione di CTC acquisisce particolare interesse perché queste cellule sono presenti nel circolo sanguigno dopo extravasazione, un fenomeno chiave del 
processo di metastatizzazione. In questo campo, numerosi studi si sono concentrati sulla valutazione della transizione epitelio-mesenchimale: la perdita di molecole di adesione tipiche delle cellule epiteliali e l'acquisizione di motilità da parte delle cellule tumorali può testimoniare la maggiore aggressività delle cellule tumorali stesse e predire un comportamento clinico aggressivo.

Frammenti di acidi nucleici (sia DNA che RNA) derivati dalle cellule tumorali si ritrovano in circolo sia per rilascio diretto da parte delle cellule tumorali che vanno incontro a necrosi o apoptosi, sia dopo la digestione da parte di macrofagi dopo fagocitosi, o per il rilascio da parte delle cellule tumorali circolanti [18]. La presenza di specifiche mutazioni può differenziare $\mathrm{i}$ frammenti di acidi nucleici di origine tumorale da quelli derivanti da cellule non neoplastiche. Questo materiale può essere utilizzato per la ricerca di specifiche mutazioni caratterizzanti una neoplasia ed è già utilizzato in ambito clinico per la valutazione dello status mutazionale del recettore EGFR nei pazienti affetti da tumore del polmone non a piccole cellule [19]. Va considerato che gli acidi nucleici, derivando prevalentemente da cellule necrotiche o apoptotiche, potrebbero non essere rappresentativi dell'eventuale porzione cellulare resistente al trattamento.

Le cellule tumorali possono rilasciare nella circolazione microvescicole, note come esosomi, contenenti proteine, acidi nucleici, lipidi e metaboliti. Questi esosomi sembrano correlare con la progressione tumorale, la risposta immune, l'angiogenesi e la metastatizzazione [20]. In particolare, la composizione delle integrine del lisosoma potrebbe predire la diffusione metastatica tumorale partecipando alla formazione della nicchia metastatica.

Infine, è stata dimostrato come l'interrelazione tra le piastrine del paziente e le cellule tumorali sia implicata nella crescita e disseminazione del tumore. Queste piastrine, che vengono quindi chiamate TEP, sono anche in grado di veicolare biomolecole associate al tumore. In particolare, le TEP hanno la capacità di fagocitare RNA circolare rilasciato dal tumore [21]. È stato dimostrato come lo studio delle TEP permetta di discriminare soggetti affetti da tumore e soggetti sani, di identificare la tipologia di tumore primitivo e di valutare dei biomarcatori che possano predire la risposta al trattamento.

Nonostante i numerosi vantaggi della biopsia liquida, al momento vi sono alcune criticità che ne riducono l'applicazione clinica. In primis non tutti gli istotipi tumorali rilasciano una pari quantità di cellule tumorali (o di altri componenti) nel circolo sanguigno, anche in caso di alto burden tumorale [22]. Questo, unito al fatto che solo una piccola percentuale di esosomi, piastrine o frammenti di acidi nucleici è di fatto di origine tumorale, potrebbe comportare una scarsa disponibilità di materiale e, quindi, una ridotta accuratezza dell'analisi $[12,13]$. Inoltre, andrebbe incrementata la standardizzazione delle procedure sia preanalitiche che analitiche. Infine, non sempre eventuali mutazioni o alterazioni evidenziate con la biopsia liquida sono dotate di un'accuratezza sufficiente da poter permettere la corretta diagnosi o da poter guidare la gestione clinica del paziente [23].

\section{NETest}

Anche nelle NEN, studi preliminari hanno dimostrato che i prodotti dell'espressione genica rilevati a livello ematico rispecchiano l'espressione genica del tessuto tumorale e che quest'ultima correla strettamente con il comportamento biologico della neoplasia in esame [24].

Partendo da questi assunti è stato elaborato il NETest che, tramite una tecnica di biologia molecolare, la real time PCR (rt-PCR), permette di verificare e quantificare nel sangue dei pazienti la presenza di diversi prodotti trascrizionali (mRNA) di origine neoplastica [25].

In particolare, si fa riferimento a un panel contenente 51 geni implicati nella tumorigenesi neuroendocrina e appartenenti alle categorie dei cosiddetti "-omi”" (SSTRoma, proliferoma, metaboloma, secretoma, epigenoma, apoptoma) che costituiscono nell'insieme il fingerprint caratteristico delle NEN [26]. Tali geni sono stati selezionati tramite un processo multistep sfruttando la tecnologia dei microarray, che consente di verificare quanti e quali geni sono attivi in uno specifico tipo cellulare o in un tessuto e qual è il loro livello di espressione. Comparando tessuti sede di NEN e di altri istotipi tumorali con tessuti non interessati da patologia, sono stati selezionati i geni iperespressi solo nelle GEP-NEN $\mathrm{e}$, tra questi, sono stati successivamente individuati quelli che si ritrovavano nel trascrittoma ematico. Integrando questi geni con quelli già noti in letteratura si è ottenuto il panel attualmente utilizzato [25].

Il NETest si articola in due parti (Fig. 2):

1. dal sangue periferico del paziente, raccolto e messo in provetta con EDTA, sono isolati gli mRNA e da questi vengono prodotte le sonde di cDNA. Viene quindi eseguita una rt-PCR che amplifica i geni di interesse;

2. si quantificano tali geni e tramite algoritmi viene realizzata una prima analisi che restituisce un punteggio da 0 a 8: campioni con un punteggio $0-2$ sono ritenuti normali, da 3 a 8 sono considerati associati a NEN. Viene poi effettuata una seconda analisi algoritmica che valuta l'espressione di precisi clusters genici: valori superiori o uguali a 50 corrispondono a una probabilità superiore al $75 \%$ di trovarsi di fronte a una malattia in progressione.

Queste due informazioni vengono sommate e convertite in un valore percentuale (da 0 a 100\%): il cosiddetto NETest score. Questo score è direttamente proporzionale al livello di attività della malattia al momento del test: $0-40 \%$ : bassa attività, 41-79\%: moderata attività, $\geq 80 \%$ : alta attività [27]. 
Fig. 2 Metodologia del netest

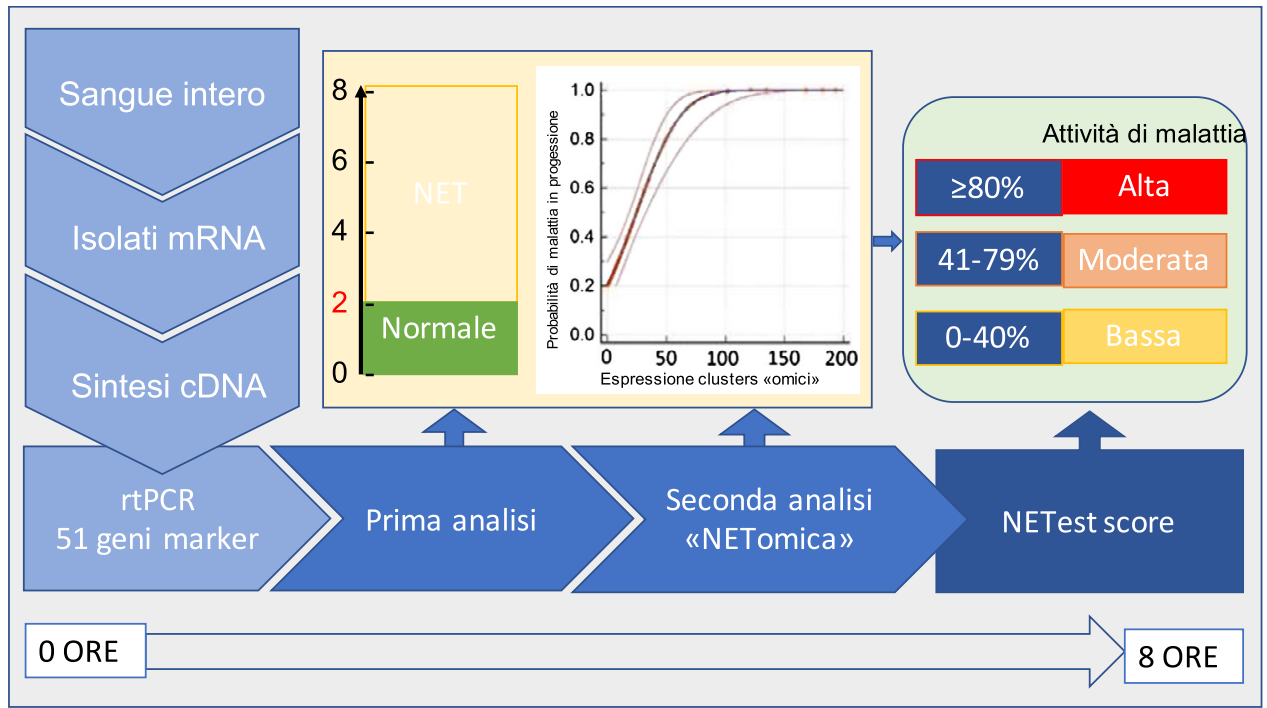

Tabella 2 Studi clinici sul ruolo diagnostico del NETest. GEP $N E T$, tumore neuroendocrino gastroenteropancreatico; PNET, tumore neuroendocrino pancreatico; NEN, neoplasia neuroendocri- na; $N E C$, carcinoma neuroendocrino; $B P C O$, broncopneumopatia cronica ostruttiva; $P P G L$, feocromocitoma e paraganglioma; $G E P$, gastroenteropancreatico

\begin{tabular}{|c|c|c|c|c|}
\hline & Pazienti & Controlli & Risultati & NETest cutoff \\
\hline $\begin{array}{l}\text { Malkzwesca A et al [31], } \\
2019\end{array}$ & -111 GEP NET & - 63 controlli sani & $\begin{array}{l}\text { - Performance diagnostica } \\
\text { Sensibilità 99\% } \\
\text { Specificità 95\% } \\
\text { Accuratezza } 97 \%\end{array}$ & - \\
\hline Genc CG et al [35], 2018 & - 35 PNET & - 11 controlli sani & $\begin{array}{l}\text { - NETest score }>\text { nei pazienti che } \\
\text { nei controlli }(p<0,05)\end{array}$ & $\begin{array}{l}>20 \% \text { diagnosi } \\
\text { di NET }\end{array}$ \\
\hline Filosso P et al [32], 2018 & $\begin{array}{l}\text { - } 131 \text { bronchopulmonary } \\
\text { NEN (carcinoidi tipici e } \\
\text { atipici e NEC) }\end{array}$ & $\begin{array}{l}\text { - } 49 \text { controlli con } \\
\text { neoplasia } \\
\text { non-neuroendocrina } \\
\text { - } 18 \text { controlli BPCO } \\
\text { - } 90 \text { controlli sani }\end{array}$ & $\begin{array}{l}\text { - Performance diagnostica } \\
\text { Sensibilità } 93 \% \\
\text { Specificità } 89 \% \\
\text { Valore predittivo positivo } 92 \% \\
\text { Valore predittivo negativo } 91 \% \\
\text { Accuratezza }>88 \%\end{array}$ & - \\
\hline $\begin{array}{l}\text { Peczkowska M et al [33], } \\
2017\end{array}$ & - 32 PPG & $\begin{array}{l}\text { - } 32 \text { controlli sani o } \\
\text { affetti da patologia } \\
\text { benigna } \\
\text { - } 32 \text { NET GEP e } \\
\text { broncopolmonari }\end{array}$ & $\begin{array}{l}\text { - Performance diagnostica } \\
\text { Sensibilità } 87,5 \% \\
\text { Specificità } 100 \% \\
\text { Valore predittivo positivo } 100 \% \\
\text { Valore predittivo negativo } 93,7 \% \\
\text { Accuratezza } 96,9 \%\end{array}$ & $\begin{array}{l}>26,7 \% \text { diagnosi } \\
\text { di PPGL }\end{array}$ \\
\hline
\end{tabular}

Questa forma di biopsia liquida viene attualmente adoperata nel Regno Unito (presso il Sarah Cannon Molecular Diagnostics Laboratory di Londra) e negli Stati Uniti (presso gli WREN Laboratories di Branford, Connecticut). I dati sono identici per entrambi i laboratori e la procedura è certificata secondo i Clinical Laboratory Improvement Amendments (CLIA) [28].

Il NETest è stato utilizzato in pazienti con NEN GEP e broncopolmonari e nei paragangliomi e feocromocitomi e presenta diverse applicazioni cliniche risultando vantaggioso in ambito diagnostico, prognostico e predittivo di risposta [29]. È un metodo standardizzato e riproducibile e non è inficiato da caratteristiche del paziente come età, genere, etnia, digiuno o uso di farmaci come gli inibitori di pompa protonica [28]. Risulta quindi più vantaggioso rispetto ai biomarcatori attualmente utilizzati ed è verosimilmente destinato ad affiancarli e poi sostituirli nei prossimi anni [30].

\section{Applicazione clinica del NETest}

\section{Ruolo diagnostico}

Il ruolo diagnostico del NETest nelle NEN è stato valutato in pochi studi clinici (Tabella 2). In una serie di 111 pazienti 
Tabella 3 Ruolo prognostico del NETest. GEP NET, tumore neuroendocrino gastroenteropancreatico; $P N E T$, tumore neuroendocrino pancreatico; $N E N$, neoplasia neuroendocrina; $N E C$, carcinoma neu- roendocrino; $P P G L$, feocromocitoma e paraganglioma; $P D$, malattia progressiva; $S D$, malattia stabile

\begin{tabular}{|c|c|c|c|}
\hline & Pazienti & Risultati & NETest cutoff \\
\hline $\begin{array}{l}\text { Malkzwesca A et al } \\
\text { [31], } 2019\end{array}$ & $\begin{array}{l}-75 \text { GEP NET } \\
11 \mathrm{PD} \\
64 \mathrm{SD}\end{array}$ & $\begin{array}{l}\text { NETest score }>\text { nei pazienti PD che SD }(p \\
<0,0001) \text { (accuratezza 95\%) }\end{array}$ & - \\
\hline $\begin{array}{l}\text { Genc CG et al [35], } \\
2018\end{array}$ & $\begin{array}{l}\text { - } 35 \text { PNET: } \\
11 \text { R0 senza recidiva } \\
12 \text { R0 con recidiva } \\
12 \text { R } 1 \text { senza recidiva }\end{array}$ & $\begin{array}{l}\text { NETest score }>\text { in pazienti con recidiva dopo } \\
\text { chirurgia radicale che nei pazienti senza recidiva ( } p \\
<0,005 \text { ) (accuratezza 83\%) }\end{array}$ & $\begin{array}{l}\leq 40 \% \text { assenza di } \\
\text { recidiva }\end{array}$ \\
\hline $\begin{array}{l}\text { Filosso P et al [32], } \\
2018\end{array}$ & $\begin{array}{l}\text { - } 19 \text { bronchopulmonary NEN } \\
\text { (12 carcinoidi tipici, } 4 \\
\text { carcinoidi atipici, } 3 \text { NEC) }\end{array}$ & $\begin{array}{l}\text { NETest score correla con lo stato tumorale } \\
\text { post-chirurgia }(p<0,001)\end{array}$ & - \\
\hline $\begin{array}{l}\text { Peczkowska M et al } \\
{[33], 2017}\end{array}$ & $\begin{array}{l}-32 \text { PPGL } \\
11 \text { PD } \\
21 \mathrm{SD} \\
-29 \text { PPGL } \\
4 \text { metastatici } \\
7 \text { multicentrici } \\
18 \text { localizzati }\end{array}$ & $\begin{array}{l}\text { NETest score }>\text { nei pazienti PD che SD }(p<0,0001) \\
\text { NETest score }>\text { nei pazienti con malattia metastatica } \\
\text { o multicentrica che in quelli con malattia localizzata } \\
(p<0,05)\end{array}$ & $\leq 53 \% \mathrm{SD}$ \\
\hline $\begin{array}{l}\text { Modlin IM et al } \\
{[34], 2016}\end{array}$ & $\begin{array}{l}\text { - } 35 \text { GEP NET: } \\
15 R 0 \text { post-chirurgia } \\
12 R 1 \text { post-chirurgia } \\
8 \text { post-ablazione non chirurgica }\end{array}$ & $\begin{array}{l}\text { NETest score si riduce maggiormente nei pazienti } \\
\text { R0 dopo chirurgia che negli } \mathrm{R} 1 \mathrm{e} \text { in quelli } \\
\text { post-ablazione non chirurgica }(p<0,05)\end{array}$ & $\begin{array}{l}\leq 47 \% \text { assenza di } \\
\text { recidiva }\end{array}$ \\
\hline $\begin{array}{l}\text { Cwikla JB et al [36], } \\
2015\end{array}$ & $\begin{array}{l}-35 \text { GEP NET } \\
10 \mathrm{PD} \\
25 \mathrm{SD}\end{array}$ & NETest score $>$ nei pazienti PD che SD $(p<0,001)$ & $<80 \% \mathrm{SD}$ \\
\hline
\end{tabular}

con GEP-NET e 63 controlli sani, il NETest mostrava un'accuratezza del $97 \%$ nella diagnosi di NET e una concordanza superiore al 90\% con l'imaging, sia morfologico (TC e RM) che funzionale (68 Ga-SSA-PET-TC) [31]. In una casistica di pazienti con NEN polmonare, comprendente carcinoidi tipici, atipici e NEC, comparati a pazienti affetti da patologia polmonare non neuroendocrina e a soggetti sani, si ottenevano un'accuratezza diagnostica solo leggermente inferiore [32]. Tali dati sono stati anche confermati in casistiche più piccole ma omogenee di NET pancreatici e di feocromocitomi e paragangliomi [33]. In quest'ultimo studio l'accuratezza diagnostica era pari al $96,9 \%$ verso i controlli sani, mentre i valori di NETscore erano comparabili tra i feocromocitomi/paragangliomi e un gruppo di controllo con NET GEP.

Questi dati sembrano quindi fortemente suggestivi dell'efficacia del NETest nell'identificare NEN di varia origine (GEP, polmonare, feocromocitoma/paraganglioma), consentendo la diagnosi differenziale con tumori nonneuroendocrini e patologie non neoplastiche.

\section{Ruolo prognostico}

Il NETest è risultato utile anche come fattore prognostico in diversi studi clinici (Tabella 3). Il ruolo prognostico è sta- to valutato approfonditamente nello studio di Peczkowska e collaboratori [33], che evidenziava come il NETest score fosse direttamente proporzionale all'estensione di malattia, sia nella popolazione di riferimento con feocromocito$\mathrm{ma} /$ paraganglioma sia in quella con GEP-NET. Valori minori sono stati riscontrati nei pazienti con malattia localizzata, elevati invece nella malattia metastatica e multifocale. Gli autori indagavano inoltre l'utilità del NETest come predittore dello stato di malattia, riportando valori significativamente più elevati nella malattia progressiva rispetto alla malattia stabile $(86 \pm 2 \%$ vs $41 \pm 5 \%, p<0,0001)$, identificando un valore soglia pari al $53 \%$ nei pazienti con feocromocitoma/paraganglioma.

L'elevata accuratezza della biopsia liquida nel definire lo stato di malattia veniva evidenziata anche nel già citato lavoro di Filosso e colleghi sulle NEN polmonari [32], che riportava valori significativamente superiori in pazienti con patologia progressiva rispetto a quelli con malattia stabile $(73 \pm 22 \%$ vs $36 \pm 19 \%, p<0.001)$. Nel 2019 Malczewska et al. [31] confermavano risultati sovrapponibili in una popolazione di GEP NET, suggerendo l'efficacia di questo indicatore prognostico indipendentemente dal tipo di NEN.

Per quanto riguarda la capacità del NETest di predire la prognosi del tumore dopo approcci terapeutici radica- 
Tabella 4 Studi clinici sul ruolo del NETest come predittore di risposta al trattamento. GEP NET, tumore neuroendocrino gastroenteropancreatico; $P D$, malattia progressiva; $S D$, malattia stabile; $O R R$, ri-

\begin{tabular}{|c|c|c|c|c|c|}
\hline & \multicolumn{2}{|l|}{ Pazienti } & \multicolumn{2}{|c|}{ Risultati } & NETest cutoff \\
\hline $\begin{array}{l}\text { Cwikla JB et al [36], } \\
2015\end{array}$ & \multicolumn{2}{|c|}{$\begin{array}{l}\text { - } 28 \text { GEP-NET trattati con SSA } \\
20 \text { PD } \\
8 \text { SD+ORR }\end{array}$} & \multicolumn{2}{|c|}{$\begin{array}{l}\text { NETest score si riduce nei responsivi e } \\
\text { aumenta nei non-responsivi (accuratezza } \\
79 \% \text { ) }\end{array}$} & $\begin{array}{l}<80 \% \text { correla con risposta } \\
(\mathrm{SD}+\mathrm{ORR}) \text { a SSA }\end{array}$ \\
\hline $\begin{array}{l}\text { Bodei L et al [37], } \\
2015\end{array}$ & \multicolumn{2}{|c|}{$\begin{array}{l}\text { - } 54 \text { GEP NET, carcinoidi bronchiali e NEN } \\
\text { a primitivo sconosciuto trattati con PRRT } \\
15 \mathrm{PD} \\
39 \mathrm{SD}+\mathrm{ORR}\end{array}$} & \multicolumn{2}{|c|}{$\begin{array}{l}\text { NETest score si riduce nei responsivi e } \\
\text { aumenta nei non-responsivi (accuratezza } \\
89 \% \text { ) }\end{array}$} & - \\
\hline Principio attivo & Nome commerciale & \multicolumn{2}{|l|}{ Casa produttrice } & \multicolumn{2}{|c|}{ Preparazione/dosaggio } \\
\hline Everolimus & Afinitor & \multicolumn{2}{|l|}{ Novartis Farma SPA } & \multicolumn{2}{|c|}{ 2,5-5-20 mg compresse } \\
\hline Sunitinib & Sutent & \multicolumn{2}{|l|}{ Pfeizer SRL } & \multicolumn{2}{|c|}{ 12,5-25-37,5-50 mg capsule rigide } \\
\hline Cisplatino & - & \multicolumn{2}{|l|}{-} & \multicolumn{2}{|c|}{$0,5-1 \mathrm{mg} / \mathrm{ml}$ concentrato per soluzione per infusione } \\
\hline Etoposide & Vepesid & \multicolumn{2}{|c|}{ Cheplapharm Arzneimittel GMBH } & \multicolumn{2}{|c|}{ 50-100 mg capsule molli } \\
\hline Temozolomide & Temodal & \multicolumn{2}{|l|}{ Merck Sharp \& Dohme BV } & \multicolumn{2}{|c|}{ 5-20-100-140-180-250 mg capsule rigide } \\
\hline Capecitabina & Xeloda & Roche Registration GMBH & & \multicolumn{2}{|c|}{$500 \mathrm{mg}$ compresse rivestite con film } \\
\hline
\end{tabular}

Per l'elenco completo dei farmaci citati e le relative schede tecniche si rimanda al prontuario dei farmaci

li, la letteratura fornisce dati relativi a diverse popolazioni di NEN. In uno studio di Modlin del 2016 [34] eseguito su 35 GEP-NET sottoposti a chirurgia o a diverse procedure di embolizzazione, gli autori misuravano i valori di NETest e di CgA al basale e dopo l'intervento. A un mese dalla chirurgia il NETest subiva una significativa riduzione (dall' $80 \pm 5 \%$ al $29 \pm 5 \%, p<0,0001$ ), a fronte di una diminuzione trascurabile della $\mathrm{CgA}$ (da 14,3 $\pm 1,6 \mathrm{U} / \mathrm{L}$ a $12,2 \pm 1,7 \mathrm{U} / \mathrm{L}$ ); gli stessi risultati si osservavano a un mese dal trattamento con procedure ablative non chirurgiche. È interessante notare come 4 degli 11 pazienti R0 (36\%) che presentavano un aumento del NETest a un mese dall'intervento chirurgico abbiano successivamente sviluppato una positività di malattia all'imaging. L'utilità del NETest nel predire l'outcome della chirurgia radicale veniva successivamente confermata nell'ambito di NET del pancreas (G1-G2) in uno studio del 2018 [35], che evidenziava la significativa superiorità di tale metodica rispetto ai criteri clinico-patologici e ai livelli ematici di CgA.

Dallo studio di Filosso e collaboratori [32] già citato possono essere estrapolati dati riguardanti la correlazione tra il NETest score e il rischio di recidiva post-chirurgia radicale nelle NEN polmonari. Valutando l'andamento dei livelli di NETest nel postoperatorio si osservava a 30 giorni dall'intervento una riduzione del $60 \%$ rispetto al basale con valori significativamente più bassi nel caso di resezioni R0 ( $p$ $<0,001)$. sposta obiettiva; $N E N$, neoplasia neuroendocrina; SSA, analoghi della somatostatina; $P R R T$, terapia radiorecettoriale 


\section{Conclusioni}

Il NETest è una metodica di biopsia liquida applicata alle NEN che è stata di recente testata sia nelle forme GEP che broncopolmonari, oltre a feocromocitomi/paragangliomi. I risultati sono estremamente promettenti, soprattutto alla luce dell'assenza di biomarcatori efficaci per questi tumori. Tuttavia, sono necessari studi prospettici e su larghe casistiche per definire con precisione l'efficacia, l'affidabilità e il definitivo ruolo del NETest nel management dei pazienti con NEN.

Funding Note Open access funding provided by Università degli Studi di Roma La Sapienza within the CRUI-CARE Agreement.

Conflitto di interesse Gli autori Antongiulio Faggiano, Valentina Di Vito, Roberta Centello, Franz Sesti, Giulia Puliani, Tiziana Feola e Elisa Giannetta dichiarano di non avere conflitti di interesse.

Consenso informato Lo studio presentato in questo articolo non ha richiesto sperimentazione umana.

Studi sugli animali Gli autori di questo articolo non hanno eseguito studi sugli animali.

Nota della casa editrice Springer Nature rimane neutrale in riguardo alle rivendicazioni giurisdizionali nelle mappe pubblicate e nelle affiliazioni istituzionali.

Open Access This article is licensed under a Creative Commons Attribution 4.0 International License, which permits use, sharing, adaptation, distribution and reproduction in any medium or format, as long as you give appropriate credit to the original author(s) and the source, provide a link to the Creative Commons licence, and indicate if changes were made. The images or other third party material in this article are included in the article's Creative Commons licence, unless indicated otherwise in a credit line to the material. If material is not included in the article's Creative Commons licence and your intended use is not permitted by statutory regulation or exceeds the permitted use, you will need to obtain permission directly from the copyright holder. To view a copy of this licence, visit http://creativecommons.org/licenses/by/4.0/.

\section{Bibliografia}

1. Kloppel G, Couvelard A, Perren A et al (2009) ENETS Consensus Guidelines for the Standards of Care in Neuroendocrine Tumors: towards a standardized approach to the diagnosis of gastroenteropancreatic neuroendocrine tumors and their prognostic stratification. Neuroendocrinology 90(2):162-166

2. Edge SB (American Joint Committee on Cancer) (2010) AJCC cancer staging manual, 7 th edn. Springer, New York

3. WHO Classification of Tumours Editorial Board (2019) WHO classification of tumours. Digestive system tumours, vol 1. WHO, Geneva

4. Pavel M, O'Toole D, Costa F et al (2016) ENETS consensus guidelines update for the management of distant metastatic disease of intestinal, pancreatic, bronchial neuroendocrine neoplasms (NEN) and NEN of unknown primary site. Neuroendocrinology 103(2):172-185
5. Garcia-Carbonero R, Sorbye H, Baudin E et al (2016) ENETS consensus guidelines for high-grade gastroenteropancreatic neuroendocrine tumors and neuroendocrine carcinomas. Neuroendocrinology 103(2):186-194

6. Chan DL, Clarke SJ, Diakos CI et al (2017) Prognostic and predictive biomarkers in neuroendocrine tumours. Crit Rev Oncol/Hematol 113:268-282

7. Ferolla P, Faggiano A, Mansueto G et al (2008) The biological characterization of neuroendocrine tumors: the role of neuroendocrine markers. J Endocrinol Invest 31(3):277-286

8. Modlin IM, Bodei L, Kidd M (2016) Neuroendocrine tumor biomarkers: from monoanalytes to transcripts and algorithms. Best Pract Res Clin Endocrinol Metab 30(1):59-77

9. Oberg K, Modlin IM, De Herder W et al (2015) Consensus on biomarkers for neuroendocrine tumour disease. Lancet Oncol 16(9):e435-e446

10. Nolting S, Ullrich M, Pietzsch J et al (2019) Current management of pheochromocytoma/paraganglioma: a guide for the practicing clinician in the era of precision medicine. Cancers (Basel) 11(10): 1505

11. Toledo SP, Lourenco DM Jr, Santos MA et al (2009) Hypercalcitoninemia is not pathognomonic of medullary thyroid carcinoma. Clinics (Sao Paulo) 64(7):699-706

12. Mader S, Pantel K (2017) Liquid biopsy: current status and future perspectives. Oncol Res Treat 40(7-8):404-408

13. Poulet G, Massias J, Taly V (2019) Liquid biopsy: general concepts. Acta Cytol 63(6):449-455

14. Siravegna G, Marsoni S, Siena S, Bardelli A (2017) Integrating liquid biopsies into the management of cancer. Nat Rev Clin Oncol 14(9):531-548

15. Pantel K, Alix-Panabieres C (2017) Tumour microenvironment: informing on minimal residual disease in solid tumours. Nat Rev Clin Oncol 14(6):325-326

16. Ashworth $\mathrm{T}$ (1869) A case of cancer in which cells similar to those in the tumors were seen in the blood after death. Med J Aust 14:146-147

17. Sundling KE, Lowe AC (2019) Circulating tumor cells: overview and opportunities in cytology. Adv Anat Pathol 26(1):56-63

18. Schwarzenbach H, Hoon DS, Pantel K (2011) Cell-free nucleic acids as biomarkers in cancer patients. Nat Rev Cancer 11(6):426437

19. Douillard JY, Ostoros G, Cobo M et al (2014) Gefitinib treatment in EGFR mutated caucasian NSCLC: circulating-free tumor DNA as a surrogate for determination of EGFR status. J Thorac Oncol 9(9):1345-1353

20. Weidle UH, Birzele F, Kollmorgen G, Ruger R (2017) The multiple roles of exosomes in metastasis. Cancer Genomics Proteomics 14(1):1-15

21. Joosse SA, Pantel K (2015) Tumor-educated platelets as liquid biopsy in cancer patients. Cancer Cell 28(5):552-554

22. Chen L, Bode AM, Dong Z (2017) Circulating tumor cells: moving biological insights into detection. Theranostics 7(10):26062619

23. Malara N, Innaro N, Mignogna C, Presta I et al (2018) La biopsia liquida nella diagnosi del carcinoma tiroideo indifferenziato. Endocrinologo 2018(19):270-272

24. Kidd M, Drozdov I, Modlin I (2015) Blood and tissue neuroendocrine tumor gene cluster analysis correlate, define hallmarks and predict disease status. Endocr-Relat Cancer 22(4):561-575

25. Modlin IM, Kidd M, Bodei L et al (2015) The clinical utility of a novel blood-based multi-transcriptome assay for the diagnosis of neuroendocrine tumors of the gastrointestinal tract. Am J Gastroenterol 110(8):1223-1232

26. Modlin IM, Drozdov I, Kidd M (2013) The identification of gut neuroendocrine tumor disease by multiple synchronous transcript analysis in blood. PLoS ONE 8(5):e63364 
27. Lewis MA, Yao JC (2014) Molecular pathology and genetics of gastrointestinal neuroendocrine tumours. Curr Opin Endocrinol Diabetes Obes 21(1):22-27

28. Modlin IM, Kidd M, Malczewska A et al (2018) The NETest: the clinical utility of multigene blood analysis in the diagnosis and management of neuroendocrine tumors. Endocrinol Metab Clin N Am 47(3):485-504

29. Modlin IM, Drozdov I, Kidd M (2014) Gut neuroendocrine tumor blood qPCR fingerprint assay: characteristics and reproducibility. Clin Chem Lab Med 52(3):419-429

30. Modlin IM, Drozdov I, Alaimo D et al (2014) A multianalyte PCR blood test outperforms single analyte ELISAs (chromogranin A, pancreastatin, neurokinin A) for neuroendocrine tumor detection. Endocr-Relat Cancer 21(4):615-628

31. Malczewska A, Witkowska M, Makulik K et al (2019) NETest liquid biopsy is diagnostic of small intestine and pancreatic neuroendocrine tumors and correlates with imaging. Endocr Connect $8(4): 442-453$

32. Filosso PL, Kidd M, Roffinella M et al (2018) The utility of blood neuroendocrine gene transcript measurement in the diagnosis of bronchopulmonary neuroendocrine tumours and as a tool to evaluate surgical resection and disease progression. Eur J Cardiothorac Surg 53(3):631-639
33. Peczkowska M, Cwikla J, Kidd M et al (2017) The clinical utility of circulating neuroendocrine gene transcript analysis in well-differentiated paragangliomas and pheochromocytomas. Eur J Endocrinol 176(2):143-157

34. Modlin IM, Frilling A, Salem RR et al (2016) Blood measurement of neuroendocrine gene transcripts defines the effectiveness of operative resection and ablation strategies. Surgery 159(1):336-347

35. Genc CG, Jilesen APJ, Nieveen van Dijkum EJ et al (2018) Measurement of circulating transcript levels (NETest) to detect disease recurrence and improve follow-up after curative surgical resection of well-differentiated pancreatic neuroendocrine tumors. J Surg Oncol 118(1):37-48

36. Cwikla JB, Bodei L, Kolasinska-Cwikla A et al (2015) Circulating transcript analysis (NETest) in GEP-NETs treated with somatostatin analogs defines therapy. J Clin Endocrinol Metab 100(11):E1437-E1445

37. Bodei L, Kidd M, Modlin IM et al (2016) Measurement of circulating transcripts and gene cluster analysis predicts and defines therapeutic efficacy of peptide receptor radionuclide therapy (PRRT) in neuroendocrine tumors. Eur J Nucl Med Mol Imaging 43(5):839-851 